\title{
Corela
}

Cognition, représentation, langage

HS-18 | 2015

La reformulation : usages et contextes

\section{Les reformulations comportant des exemples dans les cours magistraux de l'enseignement supérieur}

\section{Marie-France Roquelaure et Claudine Garcia-Debanc}

\section{(2) OpenEdition}

Journals

Édition électronique

URL : http://journals.openedition.org/corela/4066

DOI : $10.4000 /$ corela.4066

ISSN : 1638-573X

Éditeur

Cercle linguistique du Centre et de l'Ouest - CerLICO

Édition imprimée

Date de publication : 15 novembre 2015

ISSN : 1638-5748

Référence électronique

Marie-France Roquelaure et Claudine Garcia-Debanc, « Les reformulations comportant des exemples dans les cours magistraux de l'enseignement supérieur », Corela [En ligne], HS-18 | 2015, mis en ligne le 25 novembre 2015, consulté le 19 avril 2019. URL : http://journals.openedition.org/corela/4066 ; DOI : $10.4000 /$ corela.4066

Ce document a été généré automatiquement le 19 avril 2019

\section{(c) (i) (3) (-)}

Corela - cognition, représentation, langage est mis à disposition selon les termes de la licence Creative Commons Attribution - Pas d'Utilisation Commerciale - Partage dans les Mêmes Conditions 4.0 International. 


\title{
Les reformulations comportant des exemples dans les cours magistraux de l'enseignement supérieur
}

\author{
Marie-France Roquelaure et Claudine Garcia-Debanc
}

\section{Introduction}

1 A l'Université, l'exposé magistral est le principal mode de communication des savoirs (Bouchard et Parpette, 2007, 2009). Lorsque les enseignants ont à faire partager un savoir technique aux étudiants (Bouacha, 1984) ou à leur faire comprendre un vocabulaire technique nouveau (Bouchard et Parpette, 2007), les reformulations occupent une place importante. Parmi les diverses formes de reformulations (Gülich, Kotschi, 1987), l'exemplification constitue un des moyens fréquemment utilisés pour faire comprendre la terminologie propre à la discipline. La présente contribution vise à analyser les exemples utilisés par des enseignants de Sciences du langage pour faire comprendre à des étudiants de première année les fonctions du langage, telles que les définit Roman Jakobson (1963). L'analyse porte sur les enregistrements vidéo des cours de quatre enseignants. Ces enseignants utilisent différents supports pédagogiques: supports technologiques (diaporama) ou traditionnels (polycopiés et inscription au tableau). En nous appuyant sur les travaux de Coltier (1988) et en proposant une typologie enrichie des exemples, nous allons tenter de caractériser et de comprendre de quelle manière ces exemples peuvent contribuer aux processus de construction et de traitement des savoirs chez les étudiants. Nous observerons notamment comment ils sont restitués dans un échantillon de prise de notes d'étudiants.

Dans un premier temps, nous étudierons la notion d'exemplification en la resituant dans le contexte pédagogique universitaire du cours magistral (désormais $\mathrm{CM}$ ). Dans un second temps, nous aborderons la question de la signalisation des exemples à l'oral et à l'écrit. Nous procèderons ensuite à une analyse du traitement qui en est fait chez les enseignants 
plus ou moins expérimentés dans les supports technologiques (diaporamas) ou les inscriptions au tableau et dans leur discours oral. Nous essaierons enfin de voir comment ces exemples sont pris en compte dans les prises de notes des étudiants. Nous terminerons en dégageant quelques constantes utiles pour formuler des suggestions pour la formation des enseignants du supérieur.

\section{Exemples et exemplification dans des cours à l'université}

\subsection{L'exemplification : un type particulier de reformulation (Gülich et Kotschi 1987)}

Gülich et Kotschi (1987) proposent une analyse des modalités et des fonctions de la reformulation dans des interactions de la vie quotidienne ou des échanges plus techniques, tels que la consultation d'un juriste pour refuser une demande d'augmentation de loyer dans La Dame de Caluire (1987). Ils définissent la reformulation comme " une opération linguistique qui établit une relation d'équivalence entre un énoncé source (ES) et un énoncé reformulateur (ER) liés par un marqueur de reformulation (MR) » (Gülich et Kotschi, 1987 : 30). Dans cette perspective, l'exemplification est considérée comme l'une des "opérations de composition textuelle", au même titre que la définition, la dénomination ou encore le résumé. Dans la classification qu'ils proposent, elle fait partie des opérations d'expansion, dans lesquelles l'énoncé reformulateur est plus long que l'énoncé source, et plus particulièrement de la catégorie des paraphrases non définitoires. Elle est définie comme "l'apport d'éléments supplémentaires dans l'énoncé reformulateur ».

$4 \mathrm{Si}$, dans les traditions de la rhétorique, l'exemple est considéré comme un procédé argumentatif pour convaincre, constituant une preuve à l'appui de l'argument auquel il correspond, en contexte d'enseignement, l'exemplification peut être vue comme « une stratégie d'intervention cognitive » au service de la compréhension de la terminologie d'une discipline. (Nonnon, 1993 : 203). Comme le souligne Nonnon dans son article, les connaissances véhiculées peuvent être appréhendées par le biais de « situations concrètes réelles ou imaginées » et par des renvois permanents aux termes et notions évoqués (Nonnon, 1993 : 103).

\subsection{L'exemplification dans les cours magistraux}

5 A l'université, dans les cours, les exemples occupent une place importante. Le cours magistral se présente en effet comme un monologue dialogique qui met en jeu une relation dissymétrique entre un enseignant garant du savoir et des étudiants chargés de l'écouter de manière active et de retranscrire les connaissances dans leurs prises de notes. L'enseignant est rarement interrompu par des questions ou remarques des étudiants et, contrairement à ce qui se passe dans l'enseignement primaire ou secondaire, il n'organise généralement pas de questionnement des étudiants. Le discours est donc monologal. Ce discours peut être considéré comme dialogique dans la mesure où il est traversé par des citations des auteurs auxquels se réfère l'enseignant. En effet, le discours universitaire a pour finalité principale la transmission de connaissances rapportées aux auteurs de ces théories. 
6 Elaboré sur la base de différents supports pédagogiques de nature technologique (diaporama) ou de façon plus traditionnelle (polycopiés et inscription au tableau), le cours magistral a été caractérisé par Bouchard, Parpette, Pochard (2005) comme " oralographique ", dans la mesure où il fait coexister les modalités orale et écrite. En effet, l'enseignant parle en même temps qu'il projette un diaporama ou qu'il commente un polycopié. Cette caractéristique semble commune à différentes situations de travail, comme les concertations en bureau d'ingénieur, qui associent discussion orale et coprésence de documents écrits.

7 De leur côté, les étudiants doivent simultanément écouter l'enseignant et traiter cet oral pour inscrire certains éléments du savoir exposé dans leurs prises de notes. La vitesse d'écriture étant très inférieure au débit à l'oral, l'enseignant, pour faciliter la prise de notes des étudiants peut ralentir le débit, dicter certains éléments particulièrement importants ou occuper le temps en présentant des éléments moins centraux, de façon à laisser le temps aux étudiants de noter les éléments-clés.

On peut penser que l'exemplification remplit plusieurs fonctions : (a) elle rend le cours plus attrayant, (b) elle permet aux étudiants de comprendre les termes techniques que l'enseignant leur présente, (c) elle contribue à diminuer la densité des informations transmises dans le cours. (d) Ces exemples peuvent également aider l'étudiant à mieux mémoriser les notions. Nous allons voir plus particulièrement que l'exemplification est un procédé convoqué pour illustrer la terminologie du domaine. Mais on peut se demander dans quelle mesure les étudiants accordent de l'importance aux exemples et les retiennent dans leur prise de notes.

\subsection{De l'opération d'exemplification à l'exemple dans les travaux de Coltier (1988)}

Coltier (1988) propose une théorie de l'exemplification basée sur les travaux relatifs à la paraphrase entrepris par Fuchs (1982). L'opération d'exemplification est la convocation d'un « extrait du réel » qui se donne à tous et à chacun [...] cité à comparaître à des fins d'attestation" (Coltier, 1988: 24). L'auteur considère par conséquent que "tout énoncé présenté comme un énoncé exemple donne à lire que le fragment paraphrasé est le nom générique d'un ensemble, d'une classe. L'exemplification apparait donc comme une opération discursive qui consiste à établir l'équivalence de deux énoncés dont l'un est présenté comme une liste paradigmatique inachevée des individus qui composent la classe et qui sont donnés comme autant de faits ou de choses vérifiables" (Coltier, 1988: 26). Ici l'exemple est vu comme "un fragment du réel » qui atteste un savoir ou qui l'expose à l'attention du sujet. L'exemple met en jeu " une relation d'identité entre un exemplifié c'est-à-dire ce qu'il exemplifie, [...] et d'un autre côté les cas et les évènements qui sont comme lui, exemples de l'exemplifié ". (Coltier, 1988 : 23). On peut donc considérer, avec Coltier, qu' « un énoncé est un exemple à condition d'une part que le contenu qu'il dénote puisse être tenu pour un cas particulier dans l'énoncé exemplifié, à condition d'autre part que l'énoncé exemplifiant lui-même puisse être tenu pour un énoncé fragmentaire qui laisse en suspens l'énumération d'un paradigme " (Coltier, 1988: 86). Cette opération d'exemplification est caractérisée par des indicateurs sémantico-lexicaux (par exemple, prenons un exemple..) dans des relations bidimensionnelles hypéronymie/ hyponymie (générique/spécifique) et articulation classe / éléments appartenant à la classe. 


\section{Questions de recherche, méthodologie et corpus analysé}

\subsection{Questions de recherche}

Les questions de recherche auxquelles nous cherchons à répondre sont les suivantes :

- Quelle place occupent les exemples dans un cours destiné à des étudiants de première année de Sciences du langage comportant une terminologie technique?

- Peut-on déterminer différentes catégories d'exemples?

- Quelle place relative occupent les différentes catégories d'exemples dans le discours oral des enseignants?

- Ces exemples sont-ils de natures différentes chez des enseignants très expérimentés et chez des enseignants débutants?

- Comment les étudiants traitent-ils les exemples dans leurs prises de notes?

\subsection{Corpus analysé}

Le corpus sur lequel nous avons travaillé est composé de 8 cours à des étudiants en L1 de Sciences du Langage assurés à la fois par deux enseignants expérimentés et par deux chargés de cours débutants. Dans le cadre de cette contribution, nous analyserons plus particulièrement deux extraits d'environ $45 \mathrm{mn}$ chacun, dont nous joignons un échantillon en annexe. Ces deux extraits portent sur un même contenu : au sein du thème « communication verbale », l'étude de deux fonctions du schéma linguistique de Roman Jakobson, la présentation de la fonction phatique et de la fonction métalinguistique. Le premier document est extrait du cours d'un enseignant débutant, que nous appellerons Irène. Le second document est issu de la transcription du cours d'un enseignant expérimenté, que nous appellerons Maurice. Cet enseignant a plus de dix ans d'expérience et est l'auteur du cours à distance SED partagé avec l'ensemble des enseignants assurant cet enseignement. L'enseignant I utilise tableau et polycopié, tandis que l'enseignant $\mathrm{M}$ commente des diapositives qu'il a construites en reproduisant quasiment à l'identique le cours du SED.

\subsection{Conventions de transcription}

12 Nous avons transcrit les productions orales de chaque enseignant en nous conformant aux conventions de transcription du GARS Groupe Aixois de Recherche en Syntaxe (Blanche-Benveniste, 1999: 14). L'orthographe est normalisée. Les retranscriptions ne comportent donc aucun signe de ponctuation ni de majuscules, qui sont réservées aux noms propres. Les lettres capitales utilisées pour l'ensemble d'un mot permettent de traduire une intensité importante marquant l'insistance. La durée des pauses longues est indiquée entre parenthèses. Les signes XXX correspondent à des passages inaudibles.

13 Afin de faciliter la compréhension et de mettre en évidence la dimension oralographique, nous avons opté pour une représentation tabulaire, en mettant en regard la transcription des propos oraux et les supports pédagogiques écrits. Le tableau se compose de deux colonnes. La première, que l'on a nommée « diaporama » ou « tableau », présente les écrits présents sous les yeux des étudiants, qui peuvent être, suivant le cas, des appuis 
iconiques de type diaporama projetables via l'ordinateur ou des inscriptions au tableau réalisées pendant le cours. La deuxième colonne reporte l'intégralité des retranscriptions des discours de chaque séance. Elle comporte des didascalies indiquant la situation d'enregistrement, à savoir les personnes présentes et ou le ou les supports pédagogiques utilisés par l'enseignant, ainsi que des indications sur les éléments non verbaux, formulées entre parenthèses, ou l'intonation (flèches ascendantes ou descendantes en fin d'énoncé).

\section{Les exemples dans les cours: analyse qualitative}

\subsection{Typologie des exemples}

Nous avons dégagé 4 types de reformulations dont la première est une reprise des travaux de Coltier (1988). Pour chacune des catégories, nous indiquerons un exemple extrait du corpus restreint figurant en annexe.

La première catégorie s'intitule, comme chez Coltier (1988), "Exemple typique de la classe». Elle se caractérise par l'extraction d'un ou plusieurs individus typiques de la classe :

(1) I - comment est ce qu'on permet comment est-ce qu'on régule la communication et qu'on vérifie que l'autre est toujours bien là qu'il a bien compris par exemple dans_une question alors $\backslash$

004. EEE : on dit t'es toujours là 国

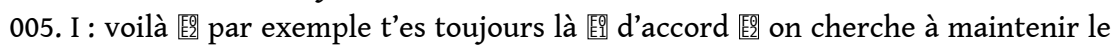
contact d'accord?

On peut remarquer une sorte de double mouvement d'exemplification, du plus abstrait au plus concret. La fonction de régulation de la communication est exemplifiée par la notion de question, elle-même exemplifiée par un exemple de question proposée par un étudiant, dans un échange dialogal. Les deux exemples, situés à des niveaux hiérarchiquement différents, par rapport à l'affirmation théorique sont tous deux signalés par le même marqueur, par exemple. Les exemples sont encadrés par des affirmations générales théoriques comment est-ce qu'on régule la communication en amont des exemples et on cherche à maintenir le contact après les exemples. La séquence d'exemplification se clôt par une recherche d'accord avec l'auditoire : d'accord?

17 A cette catégorie, nous ajoutons un deuxième type d'exemples: "Enumération d'exemplaires de la classe ", lorsque l'exemplification s'opère d'emblée sous la forme d'une série énumérative :

(2) M - les phatiques sont des interjections telles que « Hein » « n'est-ce pas » «tu vois » « allo"

Les exemples sont introduits par un marqueur linguistique telles que et figurent au nombre de quatre, de registres de langue différents.

Dans les enregistrements que nous avons analysés apparaissent fréquemment des exemples longuement développés sous la forme de récits. Nous avons, pour cela, créé deux nouvelles catégories.

La troisième catégorie : «Référence à une situation vécue de l'enseignant » se caractérise par la présence de pronoms de première personne.

(3) M - ben moi si je disais à ma fille qui a 4 ans d'expliquer la fonction métalinguistique ça serait drôle 

Elle se caractérise par la présence de pronoms de deuxième personne ou d'un nous :

(4) M - imaginez que vous ne parliez pas japonais et je vous dis " saïonara "

Il est à noter que ces différentes catégories de reformulations peuvent figurer conjointement dans le discours professoral sous la forme d'une combinaison d'exemples.

\subsection{Signalisation des exemples à l'oral}

Comme nous l'avons indiqué dans la présentation des catégories, les exemples sont le plus souvent signalés à l'oral par des marqueurs lexico-sémantiques tels que par exemple, mais aussi imaginons que..., comme dans les extraits suivants :

(5) M - donc euh ::: + le code linguistique sert à décrire tous les autres modes de fonctionnement et (... ?) c'est à peu près le seul dans le langage humain +++ (Lisant) selon Jakobson + chaque fois que le destinateur et ou le destinataire juge nécessaire de vérifier s ils utilisent bien le même code + euh :::: le discours est centré sur le code + il remplit une fonction métalinguistique (7secondes) donc ça peut aller de choses très triviales c'est par exemple qu'est-ce que tu as dit? + j'ai pas bien entendu + ce que + je veux vérifier le fait que le mot sortit va bien + je te demande ce que tu as dit + ça veut dire une partie qui est la fonction phatique + c'est-à-dire maintien de la fonction phatique + c'est-à-dire + maintien de la relation mais partie + qui est une question sur le sens du mot que tu as utilisé donc la FONCTION METALINGUISTIQUE + vous comprenez ça ? (à la classe)

Le terme métalinguistique est introduit après la définition de la notion de code : chaque fois que le destinateur et ou le destinataire juge nécessaire de vérifier si ils utilisent bien le même code + euh :::: le discours est centré sur le code + il remplit une fonction métalinguistique. Selon la typologie de Gülich et Kotschi (1987), cette opération est une dénomination, dans la mesure où la définition de la notion précède le terme technique introduit. Cette définition est une citation de Jakobson lue par l'enseignant : il s'agit donc d'une citation définitoire. Nous retrouvons ici la dimension dialogique du discours universitaire, nourri par les références aux auteurs des théories qui sont présentées. Cette dénomination est complétée par plusieurs exemples. Le marqueur linguistique typique de l'exemplification c'est par exemple signale le début d'une succession d'exemples en forme de liste énumérative. Ces exemples sont ici des énoncés de la vie quotidienne.

De même, en (6), l'enseignant I signale l'exemple par une expression linguistique ++ donc_un_exemple hein donc imaginez que vous :

(6) I - donc il faut distinguer pardon 臨 réception et compréhension <excusez moi> on peut recevoir un message sans forcément le comprendre 圈 ++ donc_un_exemple hein donc imaginez que vous que vous hum _êtes heu vous n'avez jamais touché

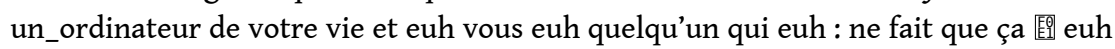
d'un point de vue péjoratif on_appelle ça XXX il sait que parler de ça et a pas forcément conscience que le champ culturel et de l'autre euh ne n'est pas partagé donc on peut tout_à fait imaginer une situation où euh l'information on va parler de son métier on va parler euh de réseau ou de choses très compliquées sans que l'autre personne ne lui parle de rien 䢻 pour autant la langue utilisée c'est le français mais comme le champ culturel n'est pas partagé la communication ne se fait pas d'accord?

L'exemple, assez longuement développé est encadré par deux affirmations générales : on peut recevoir un message sans forcément le comprendre et comme le champ culturel n'est pas partagé la communication ne se fait pas. La séquence d'exemplification est clôturée par une 
recherche d'approbation avec intonation montante, d'accord ?. Ceci met bien en évidence le fait que l'exemplification vise à impliquer l'étudiant. Cette marque est fréquente en fin d'exemple, chez les enseignants débutants et chez les enseignants expérimentés, comme on peut le voir dans l'exemple (7) :

(7) I - [...] et euh c'est_une fonction qui s'applique au langage quand_il parle de luimême 罒 (17.02) + c'est_une fonction qui s'applique au langage quand_il parle de luimême 鹋 +++ [EXED2] par exemple euh c'est euh ça permet c'est à à travers c'est_à l'aide de cette fonction qu'on va pouvoir définir un terme qui ne serait pas connu

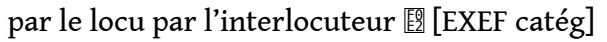

008. EEE : mais c'est la fonction métalinguistique ça?

009. I: c'est le c'est la fonction métalinguistique oui 夦 + donc imaginons une situation qui ne peut pas exister mais imaginons la 滠 je vous parle des_animaux des félins et je vous dis ben le chat hein et là 臥 vous me dites c'est quoi un chat? et moi je vous dis un chat c'est_un félin qui :: et donc dans le discours je vous_apporte une information sur un mot du lexique de manière à ce que notre champ culturel puisse coïncider 臤 (18.18) + la fonction métalinguistique c'est également aussi la possibilité de dire ah ! ben le mot chat ça s'écrit C H A T d'accord?

Dans l'extrait (7), la notion de « fonction métalinguistique » est illustrée par un exemple introduit par donc imaginons une situation. L'exemple met en scène une situation fictive dans laquelle les étudiants seraient en demande de précisions terminologiques par rapport à un terme, celui de chat, qui leur serait inconnu. Est inventé un jeu de dialogues entre les participants de la scène fictive: vous me dites c'est quoi un chat?. Le fait que l'enseignant I fasse référence dans son exemplification, à une situation didactique enseignant/étudiants n'est pas un choix anodin étant donné que la notion en question s'inscrit dans un contexte très familier pour les étudiants, la salle de cours. Toutefois, le fait que cette situation soit impossible à imaginer, puisque chacun sait ce qu'est un chat, affaiblit un peu l'exemplification et ne favorise pas la compréhension. Nous avons classé cet exemple dans la catégorie 4. Cet exemple est complété par un exemple plus court renvoyant à l'usage autonymique du langage portant sur l'épellation du mot chat. On peut noter que la marque de recherche d'adhésion d'accord n'est pas ici précédée par un retour au discours théorique, comme dans l'exemple (6) ou dans l'exemple (8) ci-dessous, extrait du discours de l'enseignant expérimenté $M$ :

(8) M - alors que euh le système d'échanges quand_on représente le schéma de la communication puisse fonctionner il faut que euh émetteur et récepteur

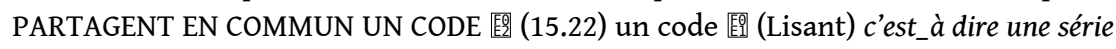
de règles qui permettent d'attribuer une signification au signe support de l'information 酸 $\mathrm{si}$ on revient au schéma de + Saussure [EXED catég 2] j'ai un signe dans ma tête + je fais une association dans ma tête + je fais une association dans ma tête et hop je l'envoie par le canal 䁂 si vous n'avez pas le même code que moi vous ne comprenez

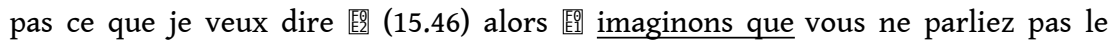
japonais et que je dis saïonara! vous vous dites mais de quoi me parle-t-il ? mais qu'est ce qu'il me dit ok ? + euh :: ça veut dire que vous ne partagez pas le même code que moi et vous vous dites il a sa façon de parler mais peut-être qu'il voulait me dire quelque chose mais je n'arrive pas à le comprendre 䢻 (16.04)+ je ne partage pas le même code que lui

L'exemple introduit par l'expression imaginons que illustre le non partage du code entre deux personnes. On note qu'il est précédé par un exemple qui fait référence au partage du code entre émetteur et récepteur. Il est à noter que, à la fin de l'exemple, l'affirmation théorique partager le même code est mise dans la bouche de l'énonciateur étudiant, indice d'une adhésion totale à l'explication donnée par le professeur. L'exemple (8) est 
développé plus longuement que l'exemple (7). On peut noter également que, comme dans l'exemple (6), les exemples sont encadrés par le discours théorique qu'ils exemplifient. (document-source du SED et diapositives), nous nous sommes appuyées sur les deux types de marqueurs dégagés dans l'étude de Mourad et Desclès (2002) portant sur l'extraction de citations textuelles: les marqueurs typographiques et les marqueurs purement linguistiques. Les marqueurs typographiques sont principalement les guillemets et les deux points. Les marqueurs linguistiques sont soit des marqueurs typiques de l'exemplification de type comme, tel que, soit des expressions spécifiques du type c'est par exemple, comme le fait de dire $X$, etc...

34 Nous avons tout d'abord analysé le document source, cours écrit du SED (Service d'Enseignement à Distance) distribué à tous les enseignants qui assurent ce cours. Voici un extrait de ce cours :

$\mathrm{Au}$ code correspond la fonction métalinguistique. Cette fonction s'applique au langage quand il parle de lui-même. Elle permet par exemple de définir des termes ignorés du locuteur (analyse du code).

Selon Jakobson: "chaque fois que le destinateur et/ou le destinataire jugent nécessaire de vérifier s'ils utilisent bien le même code, le discours est centré sur le code : il remplit une fonction métalinguistique (...) » (ibid., p. 218).

Si l'on prend l'exemple des mathématiques, il existe un langage mathématique (les nombres, les symboles algébriques) et le langage naturel pour parler des mathématiques (méta-mathématiques), alors qu'en linguistique, ou ici dans le cadre d'une théorie de la communication, nous sommes presque exclusivement limités au langage naturel pour véhiculer à la fois communication et méta-communication (cf. Une logique de la communication, p. 36). [SED] FONCTION METALINGUISTIQUE

Le terme technique à définir, en l'occurrence fonction métalinguistique, est signalé par des caractères gras. En revanche, les définitions et les exemples ne sont pas signalés par des variations typographiques. Les exemples sont signalés par des expressions 
linguistiques : par exemple, si l'on prend l'exemple des mathématiques ou par des marques de ponctuation comme les parenthèses. On peut toutefois s'interroger sur la valeur sémantique de cette parenthèse: dans un langage mathématique (les nombres, les symboles algébriques), la parenthèse encadre-t-elle des exemples ou a-t-elle une valeur définitoire? Les diapositives correspondantes de l'enseignant $M$ sont très proches $d u$ support d'origine. Ainsi, dans les diapos 7 et $8, M$ se borne à ajouter des puces et ainsi à rendre plus claire la mise en espace du texte :

[M S 2 Diapo 7] «Fonction métalinguistique »

\section{LES SIX FONCTIONS...}

\section{FONCTION METALINGUISTIQUE: LE CODE}

- Au code correspond la fonction métalinguistique.

- Cette fonction s'applique au langage quand il parle de lui-même

- elle permet par exemple de définir des termes ignorés du locuteur (analyse du code).

- Selon Jakobson :

- « chaque fois que le destinateur et/ou le destinataire jugent nécessaire de vérifier s'ils utilisent bien le même code, le discours est centré sur le code : il remplit une fonction métalinguistique (...) » (ibid., p. 218).

Dans la première diapositive [M S2 Diapo7], la définition du terme technique «fonction métalinguistique » se trouve dans un énoncé de type dénomination, où la définition précède le terme technique, comme dans le document-source du SED. Cette définition est illustrée par un exemple typique de la classe (catégorie 1), déjà présent dans le document SED. Cet exemple "définir des termes ignorés du locuteur " est introduit par un des marqueurs linguistiques de l'exemplification [par exemple] et signalé par un retour à la ligne et une puce de rang inférieur à celle qui précède la définition. Il est à noter qu'une puce identique est utilisée un peu plus loin dans la diapositive pour introduire la définition de Jakobson, dans une citation définitoire.

Une seconde diapositive est nécessaire pour présenter la suite de la définition et des exemples :

[M S2 Diapo8] « Fonction métalinguistique »

\section{LES SIX FONCTIONS...}

\section{FONCTION MÉTALINGUISTIQUE: LE CODE}

- Si l'on prend l'exemple des mathématiques,

- il existe un langage mathématique (les nombres, les symboles algébriques)

- et le langage naturel pour parler des mathématiques (métamathématiques),

- alors qu'en linguistique nous sommes limités au langage naturel pour véhiculer à la fois communication et méta-communication.

- Ainsi, seul parmi tous les systèmes de signes, le langage a un pouvoir d'interprétance, selon le mot de Benveniste.

- Seul, il peut parler de lui-même et de tous les autres langages. 

métalinguistique » est illustrée par un exemple typique de la classe (catégorie 1) puis par un exemple d'énumération d'exemplaires de la classe (catégorie 2). Cet exemple typique de la classe "l'exemple des mathématiques" est introduit par une expression linguistique [si on prend l'exemple] et signalé par une puce qui le met en relation avec la linguistique, à la puce suivante. Pour expliquer qu'on peut se servir du langage par le langage, l'enseignant choisit l'exemple des mathématiques qui constitue à son tour la classe générique à laquelle se réfèrent les exemplaires, sous forme d'énumération de liste «les nombres, les symboles algébriques », indiqués entre parenthèses. Deux puces et des caractères en italiques permettent de mettre en parallèle un langage mathématique et le langage naturel, eux-mêmes inclus dans un parallélisme signalé par des puces de niveau hiérarchiquement supérieur entre l'exemple des mathématiques et en linguistique. Les termes en italiques sont communication et méta-communication, placés ainsi en parallèle avec langage mathématique/langage naturel. On peut remarquer ainsi l'importance de la mise en espace du texte dans la diapositive et le complexité de la structure de la diapositive. diaporama sont les mêmes que ceux qui figurent dans le document commun SED. On peut noter aussi que tous les exemples de ce cours écrit sont présents dans le diaporama. diaporama, la signalisation des exemples est explicite, comme on a pu le voir dans les diapositives de l'enseignant expérimenté $M$. Les définitions des termes techniques font l'objet de nombreuses reformulations sous forme d'exemples typiques de la classe ou d'énumérations d'exemplaires de la classe. tableau au fur et à mesure du déroulement du cours, aucun exemple ne fait l'objet d'une inscription au tableau. Seuls les termes techniques sont inscrits, comme on peut le voir dans la première colonne de l'Annexe 1. différents enseignants. Pour cette étude, nous prendrons en compte les deux variables suivantes : degré d'expérience de l'enseignant et nature du support écrit utilisé. En effet, on peut penser que le niveau plus ou moins avancé des étudiants et l'expérience professionnelle des enseignants sont des paramètres qui influent sur le discours professoral et le choix des exemples. Le public d'étudiants est ici constitué d'étudiants de première année de Sciences du Langage, qui découvrent la discipline.

Nous allons procéder à une étude quantitative des différentes catégories d'exemples que nous avons dégagées en 3.1. Nous procèderons successivement à une étude d'ensemble sur l'ensemble des quatre enseignants observés puis à une étude plus détaillée portant sur les extraits figurant en annexe de cet article. 


\section{Effet des variables support et expérience professionnelle sur les types d'exemples utilisés par les enseignants.}

\subsection{Etude d'ensemble}

Nous avons tout d'abord procédé à un comptage des différentes catégories d'exemples sur l'ensemble du corpus oral.

\begin{tabular}{|c|c|c|c|c|c|}
\hline Enseignants & $\begin{array}{l}\text { Nombre } \\
\text { d'exemples }\end{array}$ & $\begin{array}{l}\text { Exemple } \\
\text { typique de la } \\
\text { classe }\end{array}$ & $\begin{array}{l}\text { Enumération } \\
\text { exemplaires de } \\
\text { la classe }\end{array}$ & $\begin{array}{l}\text { Situation } \\
\text { vécue } \\
\text { enseignant }\end{array}$ & $\begin{array}{l}\text { Situation } \\
\text { vécue } \\
\text { étudiant }\end{array}$ \\
\hline $\begin{array}{l}\text { M Expérimenté } \\
\text { Diaporama }\end{array}$ & 66 & $\begin{array}{l}12 \\
18 \%\end{array}$ & $\begin{array}{l}22 \\
33 \%\end{array}$ & $\begin{array}{l}5 \\
8 \%\end{array}$ & $\begin{array}{l}27 \\
41 \%\end{array}$ \\
\hline $\begin{array}{l}\text { L } \\
\text { Débutant } \\
\text { diaporama }\end{array}$ & 55 & $\begin{array}{l}17 \\
31 \%\end{array}$ & $\begin{array}{l}12 \\
22 \%\end{array}$ & 0 & $\begin{array}{l}26 \\
47 \%\end{array}$ \\
\hline $\begin{array}{l}\text { Z } \\
\text { Expérimenté } \\
\text { Tableau }\end{array}$ & 62 & $\begin{array}{l}10 \\
16 \%\end{array}$ & $\begin{array}{l}17 \\
27 \%\end{array}$ & 0 & $\begin{array}{l}35 \\
57 \%\end{array}$ \\
\hline $\begin{array}{l}\text { I } \\
\text { Débutant } \\
\text { tableau }\end{array}$ & $\begin{array}{l}33 \\
27 \%\end{array}$ & $\begin{array}{l}9 \\
21 \%\end{array}$ & 7 & 3 & $\begin{array}{l}14 \\
43 \%\end{array}$ \\
\hline Total & 216 & 48 & 58 & 8 & 102 \\
\hline
\end{tabular}

D'un point de vue global, nous pouvons tout d'abord noter le grand nombre d'exemples: 216 au total et plus de 50 dans tous les enregistrements sauf ceux de l'enseignant I, qui se distingue nettement de tous les autres avec deux fois moins d'exemples que l'enseignant le plus expérimenté $M$. Ces exemples sont beaucoup plus nombreux à l'oral qu'à l'écrit, puisque les diaporamas des enseignants $\mathrm{M}$ et $\mathrm{Z}$ ne comportent chacun que 6 exemples et que les enseignants $\mathrm{L}$ et I n'ont inscrit aucun exemple au tableau.

On note un nombre plus important d'exemples chez les enseignants expérimentés : 128 contre 88. Mais ce sont les faibles scores de I qui font marquer une différence importante puisque $\mathrm{L}$ utilise à peine moins d'exemples que $\mathrm{M}$ ou $\mathrm{Z}$.

La nature du support utilisé ne semble pas avoir d'incidence sur le nombre d'exemples oraux dans les enregistrements, puisque $\mathrm{M}$ et $\mathrm{Z}$ utilisent à peu près autant d'exemples. Ici encore I se distingue des autres enseignants.

Si l'on considère maintenant les différents types de catégories d'exemples, on constate que les catégories qui dominent sont la catégorie 4 « Référence à une situation vécue de l'étudiant » avec un nombre de avec 93 exemples, suivie par la catégorie 2 « Enumération 
d'exemples » avec 58 exemples, suivie de près par la catégorie 1 « Exemple typique de la classe » avec 48 exemples. Si l'on totalise catégories 1 et 2 d'une part, qui correspondent à des exemples activant plutôt la dimension conceptuelle et catégories 3 et 4 , qui font plutôt appel à une expérience affective, on obtient des scores assez proches, avec 116 exemples des catégories 1 et 2 et 110 exemples des catégories 3 et 4 . Cet équilibre est parfaitement respecté chez certains enseignants (L et I avec 29/26 et 16/17). L'enseignant M utilise davantage d'exemples des catégories 1 et 2 (44 contre 32), alors que l'enseignant 2 privilégie les catégories 3 et 4 ( 27 exemples de catégories 1 ou 2 contre 35 de catégories 3 et 4 ).

Il va s'agir maintenant de faire une analyse comparative du traitement de l'exemplification chez les enseignants expérimentés et chez les enseignants débutants en dégageant les types de catégories qui dominent à l'oral. Pour cela, nous allons analyser le poids relatif de chaque catégorie d'exemple en calculant le pourcentage que représente chacune des catégories dans le nombre total d'exemples pour chaque enseignant. La catégorie 1, " exemple typique de la classe » est mieux représentée chez les enseignants débutants (respectivement $31 \%$ chez L et $27 \%$ chez I) que chez les enseignants expérimentés ( $18 \%$ chez $\mathrm{M}$ et $16 \%$ chez $\mathrm{Z}$ ). On peut se demander si cet écart important correspond à une utilisation d'exemples isolés chez les enseignants débutants. Les exemples de catégorie 1 figurent déjà dans le document source du SED. La catégorie 2 "énumération d'exemplaires de la classe » est beaucoup plus représentée chez l'enseignant expérimenté M ( $44 \%$ des exemples) que chez ses collègues expérimenté ( 27 $\%$ chez Z) ou débutants (respectivement $29 \%$ chez L et $16 \%$ chez I). Une fois encore, I se démarque beaucoup de tous les autres enseignants. La référence à des situations vécues par l'enseignant sont plus rares chez tous les enseignants : seuls $\mathrm{M}$ et I y recourent pour moins de $10 \%$ des exemples. La référence à des situations vécues par l'étudiant est utilisée pour près de la moitié des exemples par tous les enseignants (L avec $47 \%$ des exemples, I avec $43 \%$ et $\mathrm{M}$ avec $41 \%$ ) et particulièrement par $\mathrm{Z}$, pour qui ils représentent plus de la moitié des exemples (57\%). On peut penser que ces exemples, qui renvoient à des situations plus affectives, sont destinés à capter l'attention et l'intérêt des étudiants. Leur nombre important est indéniablement lié aux caractéristiques du public, étudiants de première année, et au thème du cours, qui permet la référence à des situations de la vie quotidienne.

51 Dans le cadre de la présente contribution, nous avons choisi d'approfondir la comparaison sur un échantillon du corpus, qui figure en annexe. Il correspond à des extraits d'enregistrements des enseignants $\mathrm{M}$ et I qui, comme nous l'avons vu, présentent souvent les scores les plus contrastés. $M$ est un enseignant expérimenté, auteur du cours ; il utilise un diaporama. I est l'enseignante la moins expérimentée; elle n'utilise pas les TICE.

\subsection{Etude sur le corpus restreint}

52 Pour analyser de façon plus précise la place et la nature des exemples dans le discours de l'enseignant I et de l'enseignant $\mathrm{M}$, nous avons ajouté un certain nombre de paramètres.

Pour évaluer la longueur occupée par les exemples, nous avons compté les signes figurant dans la transcription et les signes occupés par les exemples. Le calcul que nous avons réalisé n'est pas très précis mais donne un ordre de grandeur. Nous aurions pu tout aussi bien compter le nombre de mots, avec tous les problèmes que pose une segmentation en 
mots, ou la durée effective des échanges. Ce comptage nous a permis d'évaluer le pourcentage occupé par les exemples dans le discours oral de chaque enseignant.

Il nous a paru également intéressant de regarder la longueur des exemples, pour déterminer la longueur de l'exemple le plus court et de l'exemple le plus long et comptabiliser les exemples occupant plus de 150 caractères, nombre arbitraire mais correspondant à un exemple faisant l'objet d'un développement de quelques secondes.

\begin{tabular}{|c|c|c|}
\hline Paramètres pris en compte pour l'analyse & $\begin{array}{l}\text { Enseignant } \\
\text { I }\end{array}$ & $\begin{array}{l}\text { Enseignant } \\
\text { M }\end{array}$ \\
\hline Nombre total de signes de l'extrait & 7852 & 8588 \\
\hline Nombre de signes occupés par des exemples & 1897 & 4423 \\
\hline $\begin{array}{l}\text { Place occupée par les exemples dans le discours oral : pourcentage } \\
\text { du nombre de signes }\end{array}$ & 24 & 51 \\
\hline Nombre de caractères de l'exemple le plus court & 25 & 32 \\
\hline Nombre de caractères de l'exemple le plus long & 555 & 1048 \\
\hline Nombre total d'exemples & 8 & 20 \\
\hline Nombre d'exemples occupant plus de 150 caractères & 4 & 11 \\
\hline Catégorie 1 : Exemple typique de la classe & 2 & 5 \\
\hline $\begin{array}{l}\text { Catégorie } 2 \text { : Enumération } \\
\text { exemplaires de la classe }\end{array}$ & 2 & 3 \\
\hline $\begin{array}{l}\text { Catégorie } 3 \text { : Situation vécue } \\
\text { enseignant }\end{array}$ & 1 & 3 \\
\hline $\begin{array}{l}\text { Catégorie } 4: \text { Situation vécue } \\
\text { étudiant }\end{array}$ & 3 & 9 \\
\hline
\end{tabular}

Nos observations confirment les constats précédents. Dans les deux cas, les exemples occupent une place importante dans le discours de l'enseignant et sont plus nombreux à l'oral qu'à l'écrit. Ces exemples sont beaucoup plus nombreux chez l'enseignant expérimenté M: 20 contre 8 chez l'enseignant débutant I. Le nombre de signes correspondant aux exemples est également beaucoup plus important chez $\mathrm{M}$, chez qui ils représentent $51 \%$ des signes de la transcription contre $24 \%$ pour l'enseignant I. Toutes les catégories d'exemples sont plus nombreuses chez l'enseignant $M$. C'est plus particulièrement la catégorie 4 , correspondant à des récits faisant référence à la vie de l'étudiant, qui fournit des exemples plus nombreux et plus longs dans le discours de l'enseignant $\mathrm{M}$, trois fois plus que chez l'enseignant $\mathrm{I}$. L'écart est moins important pour les catégories 1 et 2, ce qui confirme les résultats d'ensemble énoncés plus haut en 4.1.

Enfin, si les exemples les plus courts sont assez similaires dans les deux enregistrements, l'exemple le plus long est deux fois plus long chez l'enseignant expérimenté $\mathrm{M}$ et on 
trouve près de trois fois plus d'exemples longs que chez l'enseignant débutant I (11 contre 4).

Nous constatons donc un écart important entre les enseignants M et I du point de vue du nombre et du volume des exemples. Du point de vue des catégories d'exemples, l'enseignant $\mathrm{M}$ privilégie les exemples de catégories 3 et 4 (12 exemples, qui représentent $60 \%$ des exemples utilisés) par rapport à l'enseignant I (4 exemples, qui représentent 50 $\%$ des exemples utilisés).

On peut se demander dans quelle mesure les exemples sont pris en compte dans les prises de notes des étudiants.

\section{Traitement des exemples dans les prises de notes chez les étudiants des enseignants I et $M$}

\subsection{Traitement des exemples dans les prises de notes des étudiants de I}

$\mathrm{Vu}$ que le discours universitaire représente une charge cognitive assez importante dans la réception des informations à retranscrire dans les prises de notes, les étudiants mettent en place des stratégies de notations qui sont spécifiques d'un étudiant à l'autre. Voici les prises de notes de trois étudiants de l'enseignant I, par rapport au passage concernant l'introduction de la définition de la fonction métalinguistique, que nous avons cité en (7) :

[I S2 PDN 1]

Ex on peut parler d'un terme ignoré du locuteur quand on demande à l'autre : « c'est quoi un chat?»

61 [I S2 PDN 2]

Fonc ${ }^{\circ}$ métaling

- Ex : on peut définir les termes améliorés du locuteur quand on demande à l'autre : "c'est quoi un chat?»

62 [I S2 PDN 3]

[S'applique au langage qd il parle de lui-même -permet de pouvoir définir termes qui ne serait pas connu du locuteur

Ex : Qd on apporte des info ${ }^{\circ}$ sur un mot du lexique champ culturel qui coïncident

Cette fonct ${ }^{\circ}$ c'est également la possibilité de dire Ah! chat ça s'écrit CHAT]

Les prises de notes sont de longueurs très différentes. Elles attestent de la compréhension de l'énoncé professoral par les étudiants. Ainsi, la reformulation de l'étudiant 2 on peut définir les termes améliorés du locuteur montre qu'il n'a pas compris ce qui a été dit. Certains étudiants, comme l'étudiant 1 , notent littéralement ce qu'a dit l'enseignant, tandis que l'étudiant 3 abrège, ce qui lui permet de noter un plus grand nombre d'éléments. On constate, dans la majorité des prises de notes, notamment dans cette PDN 3, la présence de nombreuses abréviations de termes: qd pour quand, fonct ${ }^{\circ}$ pour fonction, info $0^{\circ}$ pour information. Chaque étudiant a ses propres conventions : ainsi pour abréger fonction, terme effectivement très fréquent dans le cours sur Jakobson, la PDN2 utilise fonc ${ }^{\circ}$ tandis que la PDN3 écrit fonct ${ }^{\circ}$. L'important est que l'étudiant puisse ensuite relire facilement sa prise de notes, même plusieurs mois plus tard, lorsqu'il fait ses révisions en préparation de l'examen. 

itoires. Seules certaines finales sont abrégées, aussi bien sur un nom commun l'info que sur un nom propre $\mathrm{Jak}_{\mathrm{k}} \mathrm{b}^{\circ}$, qu'on aurait peut-être pu abréger plus efficacement en $J$. Aucun exemple n'est noté. Notamment, l'exemple proposé par l'enseignant expérimenté $\mathrm{M}$ pour expliquer le non partage du code entre deux locuteurs, de catégorie 4, c'est-à-dire faisant appel à une situation vécue par l'étudiant, alors imaginons que vous ne parlez pas japonais et je vous dis saïonara vous vous dites de quoi me parle-t-il il ? n'a pas été repris par l'étudiant. Ce non réinvestissement de l'exemple « saïonara » peut être l'indication que sa notation n'a pas été jugée par l'étudiant utile quant à la compréhension de la notion évoquée. On peut se demander s'il a été interprété comme un exemple aidant à comprendre la notion ou comme une anecdote non essentielle dans le déroulement du cours.

7 prises de notes correspondant aux deux cours dispensés par l'enseignan expérimenté M, 8 exemples sont notés parmi les très nombreux exemples utilisés à l'oral (18). Ce sont les exemples des catégories 1 et 2 qui sont préférentiellement notés par les étudiants, comme on peut le voir dans les deux PDN ci-dessous, relatives à la définition de la fonction phatique :

\section{[M S1 PDN 3]}

Fonct $^{\circ}$ qui précède le langage articulé (nouveau-né maintient contact par gazouillemts « Allo » (flèche) directement phatique « Roger » (flèche) radio militaire

71 [M S1 PDN 4]

Phatique pour désigner les interjections telles que « hein » « tu vois », « n'est-ce pqs »... Cette $\mathrm{fct}^{\circ}$ précède le lge articulé puisq gazouillis du nvx né établit contact Ds com médiatisée il y ttes sortes de formules stéréotypées q n'ont d'autres but que de vérifier le circuit « allô » 
Il semble que les deux étudiants aient comme stratégie de noter le plus de choses possible mais ils procèdent différemment du point de vue des abréviations, très nombreuses dans la PDN 4 et très rares dans la PDN 3, qui procède plutôt par effacements syntaxiques. L'exemple de catégorie 1 concernant le gazouillis du nouveau né est noté par les deux étudiants. Pour l'exemple de catégorie 2 constitué par une énumération de quatre onomatopées, la PDN 3 ne les note pas, tandis que la PDN 4 en retient 3 sur 4 citées, les trois premières. La sélection semble liée à la vitesse d'écriture, inférieure à la vitesse de la parole orale. Elle conduit à ne conserver dans la PDN que les premiers exemples d'une énumération. Quant aux exemples illustrant des cas de communication particuliers, ils sont retenus intégralement dans la PDN 3 et commentés, alors que la PDN 4 ne retient que le premier. Devant le grand nombre d'exemples utilisés par l'enseignant $\mathrm{M}$, les étudiants opèrent une sélection. Nous avons pu constater qu'ils retiennent principalement les exemples de catégorie 1 et qu'ils abrègent les énumérations dans les exemples de catégorie 2. Il est à noter que ces exemples figurent sur le diaporama.

Bien que les données dont nous disposons soient trop limitées pour dégager des généralités, il semble que les exemples de l'enseignant I soient notés par les étudiants, alors que nous avons vu qu'ils sont souvent assez brefs, peu nombreux et ne font pas l'objet d'une inscription au tableau. Sans doute sont-ils considérés par les étudiants comme importants dans la présentation. En revanche, les très nombreux et longs exemples utilisés par l'enseignant $M$ font rarement l'objet d'une notation dans les PDN des étudiants. On peut s'interroger sur ce contraste. Plusieurs explications peuvent être proposées. On peut penser que, devant le foisonnement des exemples, les étudiants soient amenés à choisir ceux qui sont le plus parlants pour eux. Dans ce cas, la multiplicité des exemples favorise la compréhension de la notion dans le déroulement du cours, sans que les étudiants éprouvent la nécessité de les noter. Il est possible aussi que certains des ces exemples ne soient pas forcément correctement interprétés par les étudiants comme des éléments importants de l'explication. Enfin, la multimodalité, puisque les étudiants ont à la fois à lire le diaporama, à écouter le professeur et à prendre des notes ne favorise pas l'attention. On peut d'ailleurs noter que, dans l'ensemble, les exemples des enseignants n'utilisant pas le diaporama sont mieux notés par les étudiants. Mais il faudrait vérifier ces premiers résultats sur un échantillon plus important.

Les résultats le plus importants ici sont qu'on observe une très grande diversité de stratégies de prises de notes et que la notation d'exemples par les étudiants ne semble pas directement liée à leur longueur ou à leur structure dans le discours oral de l'enseignant.

\section{Conclusion}

Occupant une place primordiale dans l'enseignement supérieur, l'activité métalexicale s'avère très utilisée dans la transmission des connaissances chez les enseignants expérimentés et chez les enseignants débutants. En effet, c'est à travers elle que les étudiants peuvent approcher la terminologie d'une discipline. Motivé par l'objectif de transmettre les connaissances, l'enseignant a à sa disposition l'opération d'exemplification. Cette opération d'exemplification, qui consiste à insérer un certain nombre d'exemples, peut se réaliser sous la forme de reformulations différentes tant dans les caractéristiques linguistiques qu'elles revêtent dans le discours que dans les fonctions qu'elles assurent dans le cours. 

les enseignants débutants que chez les enseignants expérimentés. Nous avons pu voir que deux catégories d'exemples sont utilisés par tous les enseignants et notées scrupuleusement par les étudiants : ce sont les exemples typiques de la classe (Catégorie 1), qui se caractérisent par une extraction d'élément appartenant à une classe et les exemplaires de la classe sous forme de liste énumérative (Catégorie 2). Ces catégories d'exemples sont destinées à faire comprendre et à faire retenir les notions et les termes techniques inhérents à la discipline. On constate également la présence d'un grand nombre d'exemples à connotation plus affective, dans la mesure où ils font référence à une situation vécue par l'enseignant (catégorie 3) ou par l'étudiant (catégorie 4), tout particulièrement dans les discours des enseignants expérimentés. Ces exemples sont moins fréquemment notés par les étudiants. Le nombre trop limité d'enseignants observés ne nous permet pas de dégager des conclusions sur les effets de l'expérience professionnelle sur l'utilisation des exemples mais ceux-ci semblent généralement plus nombreux chez les enseignants les plus expérimentés. Cependant, le nombre d'exemples est très différent dans les enregistrements des deux enseignants débutants observés, l'un d'entre eux étant proche des enseignants expérimentés. oraux ne diffère pas sensiblement dans les divers enregistrements analysés. On peut toutefois noter que dans la modalité diaporama, un certain nombre d'exemples, principalement de catégories 1 et 2, figurent sur le diaporama et sont donc présentés aux étudiants conjointement à l'oral et à l'écrit. En revanche, les enseignants n'utilisant pas les diaporamas n'inscrivent pas d'exemples au tableau: ils réservent le tableau à la notation des termes techniques et des noms d'auteurs cités, de sorte que les exemples apparaissent alors exclusivement à l'oral. Dans les données que nous avons collectées, les exemples sont notés plus exhaustivement par les étudiants dans ce cas que dans le cas d'utilisation de diaporama. Toutefois, les données dont nous disposons ne sont pas suffisamment importantes pour confirmer ce résultat, qui demande des études complémentaires. exemples pour faire comprendre des notions difficiles à des étudiants de première année. Cette expertise est présente non seulement chez les enseignants expérimentés mais aussi chez les enseignants débutants. Elle se manifeste notamment par une signalisation des exemples par des marqueurs linguistiques au début et à la fin de l'exemple et par une structure explicite hiérarchique permettant d'articuler l'exemple à l'élément notionnel qu'il illustre. Exemples et définitions, notamment sous la forme de citations définitoires alternent dans le discours.

Nous n'avons pu collecter de données complémentaires pour évaluer l'efficacité des exemples pour la compréhension et la mémorisation des notions par les étudiants. Ainsi, nous n'avons pas pu accéder, comme nous le souhaitions, aux copies des étudiants pour analyser leur compréhension des notions et la mémorisation du cours, en regard de leurs prises de notes. Nous n'avons pu non plus nous entretenir avec les étudiants pour savoir comment ils perçoivent l'utilité des exemples dans ces cours.

Dans la perspective de formation des enseignants du supérieur, il nous parait important de prendre en compte ces observations et ces analyses pour aider les enseignants du supérieur débutants à être plus conscients de la place qu'ils réservent aux exemples, du 
traitement qu'ils en font dans le déroulement de leurs cours et de l'aide qu'ils peuvent apporter aux étudiants pour la prise de notes.

\section{BIBLIOGRAPHIE}

BLANCHE-BENVENISTE C., JEANJEAN C. (1987). Le français parlé : transcription et édition, Paris, Didier Erudition.

BOUACHA A. (1984). Le discours universitaire. La rhétorique et ses pouvoirs, Bern/Frankfurt/New York, Peter Lang, Collection Sciences pour la communication

BOUCHARD R., PARPETTE C., \& POCHARD J.-C., (2005), Le cours magistral et son double, le polycopié : relations et problématique de réception en L2. dans Pugibet, V., et Gettliffe-Grant N. et al. (eds) Pluralité des langues et des supports : descriptions et approches didactiques, Cahiers du Français Contemporain, Lyon, ENS Editions

BOUCHARD R., PARPETTE C., (2007) Initiation à une discipline universitaire, commentaire terminologique général et enseignement du vocabulaire spécialisé / Le cas des CM de Droit. In E. Calaque (Ed,) Enseignement et apprentissage du lexique. Bruxelles : De Boeck, p. 1999-211.

COLTIER D., (1988) Introduction et gestion des exemples dans les textes à thèse, Pratiques, $\mathrm{n}^{\circ} 58$, p.23-41.

FUCHS C., (1982). La paraphrase, Paris, PUF.

GULICH E., et KOTSCHI T., (1987), Les actes de reformulations dans la consultation La dame de Caluire, P. Bange, Bern, Peter Lang, p.15-81.

JAKOBSON R. (1963). Essais de linguistique générale, vol. I et II, Paris, Minuit.

KARA M., (2004) Reformulation et polyphonie, Pratiques 123-124, Metz, CRESEF, p.27-54.

MOURAD G., et DESCLES J.-P., (2002) « Citation textuelle : identification automatique par Exploration Contextuelle » Faits de Langues, n¹9, p. 179-189.

NONNON E. (1993) «" Prenons un exemple " : recours aux cas particuliers et problèmes d'intercompréhension dans les interactions didactiques ", In Halté J.-F. (coord). Interactions : actualité de la recherche et enjeux didactique, Metz, CRESEF, Université de Nancy Metz, p.201-245.

\section{RÉSUMÉS}

A l'Université, l'exposé magistral est le mode de communication principal (Bouchard et Parpette, 2007, 2009). Les reformulations y occupent une place importante. Parmi les diverses formes de reformulations (Gülich, Kotschi, 1987), l'exemplification constitue un des moyens fréquemment utilisés pour faire comprendre la terminologie propre à la discipline. La présente contribution vise à analyser la place des exemples dans les enregistrements vidéo des cours de quatre enseignants s'adressant à des étudiants de première année de Sciences du langage pour leur transmettre un même contenu. Les enseignants utilisent différents supports pédagogiques: supports technologiques (diaporama) ou traditionnels (polycopiés et inscription au tableau). En 
nous appuyant sur les travaux de Coltier (1988) et en proposant une typologie enrichie des exemples, nous allons tenter de caractériser et de comprendre de quelle manière ces exemples peuvent contribuer aux processus de construction et de traitement des savoirs chez les étudiants en observant notamment quelle place ils leur accordent dans leurs prises de notes.

At University, lectures are the primary mode of communication (Bouchard and Parpette, 2007, 2009). Reformulations are numerous. Among the various types of reformulations (Gülich and Kotschi, 1987), exemplification is a means frequently used to make students understand the terminology employed in the discipline. This paper aims to analyse exemplification in videotapes of four teachers teaching first year Linguistics students to convey the same course content. Teachers rely on different teaching materials : technological media (PowerPoint) or traditional handouts and use of the blackboard. Based on the work of Coltier (1988), we try to characterize exemples and understand how these exemples can contribute to the construction and processing of knowledge for students through their note taking.

INDEX

Mots-clés : discours enseignant, reformulations, exemples, exemplification, enseignement supérieur

Keywords : discourse of teachers, higher education, teachers

\section{AUTEURS}

MARIE-FRANCE ROQUELAURE

CLLE-UMR 5263

CLAUDINE GARCIA-DEBANC

CNRS \& UT2-Jean Jaurès 